\title{
UNA EDICIÓN COMENTADA DEL EVANGELIO DE SAN LUCAS DE PRINCIPIOS DEL SIGLO IX
}

La costumbre medieval de reutilización de viejos manuscritos que, por los motivos que fuera ', habían perdido su valor a la vista de sus propietarios, ha legado a los estudiosos un elevado número de restos de libros hoy desaparecidos, que constituyen testimonios de gran valor sobre nuestro pasado cultural. Tal es el caso de una hoja suelta de pergamino que apareció pegada a la cubierta de madera de un códice musical, ${ }^{2}$ que fue adquirido hace años por mi padre en una «librería de viejos» de Madrid. La encuadernación del manuscrito se encontraba en malas condiciones cuando llegó a mis manos, por lo que fue sometido a restauración en el antiguo Instituto de Conservación y Restauración de Obras de Arte, en el proceso de la cual se despegó el pergamino de la madera que lo sujetaba. "Puede constatarse, por restos de tinta que se observan a simple vista, que en la otra tapa del códice también existió una hoja semejante, que no llegó hasta nosotros.

El folio conservado, que está dispuesto en tres columnas, contiene un fragmento del evangelio de San Lucas (Lucas 6, 8-19), en el centro, y comentarios al texto principal, en los laterales, copiados en escritura de módulo menor. Estos van acompañados de signos de llamada que se repiten en la columna central en el lugar al que corresponden (láminas 10 a 13). Por su escritura y disposición de texto este ejemplo puede datarse a principios del siglo IX, en su segunda o tercera década, y situarse en algún escritorio del oeste alemán.

El pergamino nos ha llegado en buenas condiciones considerados los avatares por los que ha pasado. El lado del pelo ha resultado la parte más sufrida por ser la que se encontraba pegada a la encuadernación de madera. Algunos restos de su tinta han permanecido en ésta después de la restauración.

1 Una relación de los mismos puede verse en PELLEGRIN, Elisabeth, Fragments et membra disiecta, «Codicologica» 3 (1980), Leiden, p. 70-71. trabajos

2 Este códice, probablemente del siglo XIV, será objeto de uno de nuestros próximos

${ }^{3}$ La restauración se llevó a cabo cuando el citado Instituto residía todavía en el Archivo Histórico Nacional. Conste aquí nuestro agradecimiento a $D^{a}$ Carmen Crespo Nogueira, entonces directora del Centro, por la ayuda prestada. 
El folio presenta abundantes manchas, probablemente debidas a la cola, pues predominan en el lado pelo, y a humedades. No afectan excesivamente la lectura del texto principal, aunque sí a las notas laterales y al pautado, que casi no se aprecia en muchos lugares. Una doblez artificial que debió sufrir la hoja en el pasado (columna izquierda, lado carne) empeora tales circunstancias.

El pergamino es de color amarillento, más oscuro en el lado pelo. En este factor ha influido la cola con la que se pegó a las tapas del códice. La tinta es marrón muy oscuro en el lado pelo y más tenue en el lado carne, en el que en ocasiones clarea bastante.

La hoja no nos ha llegado completa pues fue cortada para adaptarla a las medidas del códice donde se reutilizó. No es posible apreciar ninguna perforación por lo que puede interpretarse que la hoja fue cortada por sus cuatro lados. Algunos pequeños rotos que se observan parecen más bien defectos del pergamino, aunque parece verse una perforación en forma de $\mathrm{V}$ del tipo de las descritas por el profesor Gilissen. ${ }^{4}$ Los cortes han mutilado por su parte superior a las tres columnas, a la primera de comentarios en su margen derecho (cara pelo) y a su opuesta en el lado carne.

El folio que ha quedado mide $247-245 \times 180-186 \mathrm{~mm}$.

La caja de escritura de la columna central tendría aproximadamente $230 \mathrm{x}$ $66 \mathrm{~mm}$, aunque la escritura con frecuencia sobrepasa estos límites. He calculado la altura de la caja considerando que en la parte superior del pergamino faltan dos líneas, según se demuestra en la transcripción que figura más adelante. En total la columna central tendría 27 líneas. Cada una de éstas mide aproximadamente $8,5 \mathrm{~mm}$ de altura.

Las columnas de comentarios tienen unos $46-48 \mathrm{~mm}$ de anchura. Su altura es difícil de calcular aunque su medida no estaría muy lejana de los 245 $\mathrm{mm}$. Tienen un número de líneas muy variable pues en ellas existen espacios en blanco que separan párrafos. En la cara pelo se cuentan 50 en cada columna y en la cara carne 58 en la primera y 48 en la segunda. A estos números habría que añadir unas 5 líneas que calculo son las que faltan en el folio por la mutilación de su parte superior. Las dos últimas líneas de la primera columna, lado pelo, se extienden por debajo del texto principal. A cada línea de texto principal equivalen algo más de dos de comentarios.

Uno de los aspectos más interesantes del fragmento que nos ocupa es el pautado combinado con la disposición del texto. Dadas las condiciones de la hoja resulta difícil ver las líneas trazadas a punta seca, que aparecen en el lado carne. Aun así, se observa que la columna central está flanqueada por una doble línea vertical ( $9 \mathrm{~mm}$ entre ambas) que actúa de separadora de los

4 Prolegomènes à la Codicologie, Gante 1977, pp. 105 y ss. Está situada en el ángulo superior izquierdo, lado pelo. 
comentarios. ' Las líneas de la columna central se apoyan también sobre un rayado horizontal a punta seca, pero no así las de las columnas de glosas que, debido a esta causa, tienden a desviarse hacia arriba. Por último, delante de la columna derecha, siempre en el lado carne, ${ }^{6}$ se aprecian, con ciertas dificultades, dos líneas separadas entre sí por $1 \mathrm{~mm}$. Ambas tienden a juntarse conforme se acercan a la parte superior de la hoja y en los tres o cuatro centímetros superiores sólo es posible ver ya una de las dos. Creo que este doble pautado parcial se debe a un error de quien preparó el pergamino para la escritura, que trazó una linea excesivamente torcida y tuvo que rectificar con otra mejor emplazada.

Puede verse el pautado esquematizado en la lámina 4 que acompaña al texto. Las líneas discontinuas corresponden a pautado y tamaño mínimo hipotéticos de la hoja.

Contamos con un interesante estudio sobre ediciones glosadas y comentadas que aclarará algunos de los aspectos de nuestro pergamino. ' $\mathrm{Ya}$ en la Antigüedad se confeccionaron libros destinados a recoger un texto principal y su comentario o, en otras palabras, códices en los que se reunían dos libros: el que contenía un texto principal y el de su comentario. El ejemplar más antiguo de la época carolingia conservado ${ }^{8}$ pertenece al $\mathrm{s}$. VIII y contiene como texto principal Ezequiel y como comentarios fragmentos de una célebre homilía de Gregorio. Escrito en minúscula irlandesa, es datable entre los siglos VIII y IX y procede de Irlanda. Presenta un pautado (lámina 1) sin duda conectado con el del ejemplo aquí estudiado. La diferencia principal entre ambos radica en que las columnas laterales presentan pautado idéntico a la central en el que se copian dos líneas en pequeño módulo de escritura en cada espacio interlineal. Este ejemplo supone un sólido apoyo al origen anglosajón de las ediciones comentadas, pero no se puede excluir una influencia del continente, de donde proceden todas las demás conocidas.

En este lugar habría que situar nuestro ejemplo, como uno de los primeros conocidos de Biblia carolingia comentada. Desde mediados del siglo IX se conocían salterios con dos columnas de glosas, éstas con doble número de líneas

S Sobre la función que ha tenido este tipo de pauta ver Renato RAFAELLI, La pagina e il testo. Sulla funzione della doppia rigatura verticale nei Codices Latini Antiquiores, Atti del Convegno Internazionale 'Il libro e il testo', Urbino 1985, pp. 1-24; y Cesare Questa, Il metro e il libro, ibidem, pp. 337-396.

- El lado pelo está demasiado deteriorado en este lugar para poder apreciar algún detalle del pautado.

'Louis HOLTZ, Les manuscrits latins à gloses et à commentaires de l'Antiquité à l'époque carolingienne, en Atti del convegno cit., pp. 139-167.

8 Zurich. Staatsarchiv, AG 19 N. ${ }^{\circ}$ XII; LOWE, CLA, VII, 1004; descrito en HOLTZ, pp. 156-7. Lámina 1. 
que el texto central, ' pero sólo más adelante, en el siglo XI, proliferan textos del Nuevo y Antiguo Testamento dispuestos según un pautado similar. ${ }^{10} \mathrm{Sin}$ embargo, la mayoría de las ediciones comentadas carolingias del siglo IX conocidas no tienen relación con la Biblia sino con el trivium y quadrivium: textos de poetas clásicos, en especial Virgilio, y gramaticales (los más antiguos, Bernensis 165, del 820, lámina 2; y Bernensis $172+$ Parisinus lat. 7929, hacia el 850 , lámina 3).

Sin embargo, aún en el s. IX, debido a las dificultades que presentaba el latín virgiliano, se continuaba leyendo sus obras en dos libros: por un lado el texto y por otro los comentarios, necesarios para la comprensión del mismo. Por ello los ejemplos del párrafo anterior hay que considerarlos casos aislados precursores del sistema de comentarios. En concreto, Holtz opina que el 172 reproduce la forma primera en la que fueron reunidos escolios a partir de los comentarios autónomos según modelos insulares. ${ }^{12}$ ¿Es lícito aplicar este modelo y proceso a los textos sagrados? Dado que contamos con escasos ejemplos del siglo IX, y sólo uno de principios, el aquí estudiado, me inclino a responder de forma afirmativa.

Los tres ejemplos reproducidos en las láminas 1 a 4 tienen un evidente parecido (columna central y simétricas laterales separadas por espacios libres verticales), aunque también presentan diferencias:

- El pautado de las columnas laterales es diferente: ancho, en el que caben dos líneas de pequeño módulo (Zurich); estrecho, para una línea de escritura de pequeño módulo (Berna); sin pautado, ${ }^{13}$ por lo que las líneas se desvían hacia arriba, en el cuarto ejemplo.

- La proporción entre la anchura de las columnas central y laterales difiere en los tres (Zürich 74:69 = 1.07; Berna 172 88:70 = 1.25; Madrid $66: 46=1.43$ ó $66: 48=1.37$ ). El Bernensis 165 (lámina 2) presenta, como el

9 Por ejemplo Frankfurt/M. Barth 32, de Fulda, primera mitad del s. IX, citado por Bernhard BISCHOfF, Paléographie de l'Antiquité Romaine et du Moyen Age Occidental, París 1985 , pp. 36 y nota 72 , donde da entender que existen más ejemplos sin aludir a su procedencia ni datación.

10 HolTZ, cit., pp. 157 y nota 55.

1 Un ejemplo procedente de Tours, de mitad o segunda parte del IX, pude verse en Elisabeth PELLEGRIN, Les manuscrits classiques latins de la Bibliothèque Vaticane, Paris 1982, pp. 379 y ss., con una lámina: Pal. lat. 1710; otros ejemplos de mitad del IX en Cesare Questa, cit., Tav. 24a, Paris, Bibl. Nat., lat. 7972; algo anterior 24b, Leiden, Bibl. der Rijksuniversiteit, Bibl. publ. lat. 28.

12 HolTz, cit., pág. 159 y 160-1.

13 ¿A qué se debe esta falta? Es posible que a evitar un exceso de trabajo por la escasa utilidad de este pautado: las líneas son muy cortas y no es necesario. Las columnas de comentarios de este ejemplo apoyan esta idea, pues son bastante más cortas que en los demás. 
cuarto ejemplo, columnas laterales mucho más estrechas que la central (120:55 $=2.18$ !), siendo ambos de datación similar (820 c.).

- El cuarto ejemplo es de forma clara el de menor tamaño.

A pesar de estas diferencias es obvio que todos los ejemplos guardan parecido entre sí y que ninguno de ellos puede ser el resultado de una improvisación: tienen una tradición sólida ya canonizada.

En la introducción en el continente de la edición comentada en época carolingia confluyen, según Holtz, tres elementos:

- Existencia de modelos glosados procedentes de la Antigüedad.

- Necesidad de unir en un solo libro texto y comentario interpretativo.

- Necesidad de unificar y simplificar, de facilitar el acceso a generaciones menos cultas del material complejo legado por los clásicos.

De nuevo hay que preguntarse si esta misma necesidad pedagógica que dio origen al nuevo tipo de libros se produjo también en textos bíblicos. El Ezequiel de Zürich y el ejemplo que publicamos parecen ser las pruebas confirmatorias. El primero, como ejemplar más antiguo conservado de una edición comentada, sería una adaptación a la literatura sagrada de un estilo de edición procedente de textos profanos. El segundo ${ }^{15}$ representa la consagración del sistema en textos sagrados en tiempos tan tempranos como los inicios del siglo IX. La densidad y regularidad ${ }^{16}$ del texto de sus comentarios permite afirmar que no debe ser el primer ejemplo de la tradición sino que probablemente siga un modelo compuesto con anterioridad.

En general el manuscrito carolingio contiene muchas glosas, notas y escolios, más que los antiguos. Ello se debe a que el libro carolingio tiene un público diferente que el antiguo. El hombre del s. IX es un escolar que necesita en un libro a la vez el texto a estudiar y la clave para comprenderlo. Si esta clave no existe, el s. IX la compone, ${ }^{18}$ tanto en textos profanos como sagrados.

${ }_{14}$ Beryl SMalley, The study of the Bible in the Middle ages, Oxford 1984, capítulo II, teoriza el proceso de canonización de las glosas aunque sin proporcionar ejemplos gráficos.

1s Junto con los ejemplos más antiguos citados por BISCHOFF (ver nota 9).

16 Sólo en la cara pilosa en las dos últimas líneas el copista abandona el pautado de la columna primera, aunque espacio para continuar más abajo no le faltaba. También hay que decir que en tres de las cuatro columnas de comentarios se sobrepasa la línea inferior horizontal del pautado.

17 Las PL. 5 (Berna 172) y 6 (Berna 165) de HolTZ muestran una extrema irregularidad en los comentarios, que presentan muchos espacios en blanco y copia a veces confusa. Lo mismo ocurre con los ejemplos de la nota 11. Más compacto es el Zürich de las PL. 3 y 4 . ¿Significa ello que los comentarios bíblicos son más avanzados y elaborados que los de textos profanos?

18 Holtz, cit., pág. 166.

19 Otfrid von Weissenburg - ver nota 22- escribe sus comentarios a la Biblia para 
Por su escritura este fragmento puede datarse en la primera mitad del siglo IX y, más concretamente, en su segunda o tercera década, y situarse en algún escritorio de la Alemania occidental. ${ }^{20}$ La doble forma de la $a$, la presencia de nexos cursivos, de $N$, la $g$ sin ojo son, entre otras, características habituales de este tiempo. ${ }^{21}$ El origen alemán de algunos de los primeros ejemplos comentados que conservamos contribuye a apoyar esta hipótesis.

El aspecto de la escritura de las dos caras de la hoja es algo diferente. La del lado pelo es más regular, gruesa y de mayor módulo que la del lado carne que, a simple vista, resulta mucho más sutil y de trazado vacilante. Entre ambas también hay pequeñas diferencias morfológicas. Valga esto tanto para la columna central como para las de comentarios.

La escritura en general es pesada, inclinada hacia la derecha, ${ }^{23}$ en los comentarios de forma más acentuada. Estos últimos son de trazado más irregular debido a la dificultad de ejecución de letra de pequeño módulo. Es conocida la tendencia carolingia (siglos IX al XII) hacia la distinción de la escritura del texto de la de los comentarios, no sólo en su tamaño, sino también en la forma de las letras. ${ }^{24}$ En el texto central las letras adoptan mayor número

lectores que debían tener a la vista, o por lo menos en la mente, el texto latino. Sin éste, el texto del comentario con frecuencia carece de sentido.

${ }^{20}$ Debemos estos datos al Prof. Dr. Bernhard Bischoff, a quien testimoniamos cordialmente su experta e inestimable ayuda. La compleja división en provincias escriptorias (Schriftprovinzen) de época carolingia puede verse en su trabajo Centri scrittorii e manoscritti mediatori di civiltà dal VI secolo all'età di Carlomagno, en G. CAVALLo, Libri e lettori nel medioevo, Roma-Bari 1989, pp. 29-72.

${ }^{21}$ Hans BuTZManN, Die Wolfenbüttler Fragmente der Historien des Gregor von Tours, «Scriptorium» XX (1966) pp. 31-40, y láms. 2-8, sobre todo la 2, también procedente de una hoja de guarda, similares a mi ejemplo. Escrituras alemanas pueden verse en $B$. BISCHOFF, Die sïdostdeutschen Schreibschulen und Bibliotbeken in der Karolingerzeit, I, Die Bayrischen Diözesen, Leipzig 1940, y II, Die vorwiegend Österreichischen Diözesen, Wiesbaden 1980; también sobre Baviera, del mismo autor, Kalligraphie in Bayern. Achtes bis zwölftes Jabrhundert, Wiesbaden 1981.

${ }^{22}$ En Weissenburg un discípulo de Rábano Mauro que estudió en Fulda entre el 820 y el 830, Otfrid, dirigió la copia de glosas bíblicas en lengua germánica que él mismo completó con notas marginales (entre 865 y 870), BISCHOFF, Paléographie (cit.), pp. 133; Donald A. MACKENZIE, Ottfrid von Weissenbur g: narrator or commentator?, Stanford University Press, 1946; Mittelalterliche Studien, III, Paläographische Fragen deutscher Denkmäler der Karolingerzeit, pp. 76-77. Ver además n. 9.

${ }_{23}$ Una posible influencia de Tours debe tenerse en cuenta aquí, pues de este escritorio se enviaron en el s. IX Biblias caligrafiadas a numerosas abadías e iglesias (BISCHOFF, Paléograpbie, pp. 129).

${ }_{24}$ "Glossenschrift» de B. BISCHOFF, descrita en La nomenclature des écritures livresques du IXe au XIIle siècle, París 1954, pp. 8; aquí se distinguen a, con doble forma en texto y una sola en glosas; $r$ y algunos astiles altos en ocasiones más largos en glosas, etc. 
de formas y conservan más elementos cursivos. Los comentarios son más cercanos a la carolina aunque la uniformidad de esta escritura todavía no ha acabado con las reminiscencias antiguas. Abordamos la descripción morfológica de letras, nexos y demás signos gráficos.

MAYÚSCULAS. - Las del lado pelo son más gruesas de forma ostensible. $N$ mayúscula aparece en ambas caras dentro de la línea, en posición inicial o después de signo de puntuación. Una a minúscula de módulo mayor aparece en esta circunstancia en cara carne, columna izquierda, apostoli (línea 33). El nexo et adopta a veces módulos mayores que las minúsculas. $F$ e $I$ dividen su pie en su parte inferior ${ }^{25}$ (lámina 5 ).

MINÚSCULAS.- Dadas las semejanzas entre ambas caras de pergamino, su escritura se comenta de forma conjunta.

a Adopta tres formas diferentes en el texto central -doble $c$ abierta de dos puntas («Spitzen»), doble $c$ cerrada y típica carolina- mientras que en los comentarios sólo aparece la tercera de las mismas. La espalda de esta forma se inclina hacia la derecha. A final de palabra se alarga la forma abierta.

$b$ El ojo nunca cerrado del todo en lado pelo. En los comentarios más inclinado a la derecha que en el texto central. Astil de altura variable en lado carne.

$e$ Su trazo medio toca la letra siguiente y al final de línea o palabra suele alargarse. El punto de ataque del trazo inferior destaca con frecuencia. Otras veces la letra es totalmente redonda. Forma bastantes nexos. Alguna caudata en los comentarios.

$f$ Su trazo medio tiende a tocar la letra siguiente. El superior aparece a veces destacado.

$g$ En el texto adquiere aspecto semiuncial pero con ductus claramente carolino, abierta arriba y abajo (forma de 3 con trazo de enlace con la siguiente letra). En los comentarios se confirma este detalle. Suele tocar o formar nexo con la letra siguiente.

$b$ En comentarios en ocasiones vertical.

i Siempre corta. Sólo a principios de la línea quinta, cara pelo, aparece un ejemplo más largo.

$l$ Curva bien marcada por la que toca la letra siguiente.

$m n$ La última pata inclinada tanto hacia adentro como hacia afuera. En ocasiones más corta que las restantes.

- En comentarios unas pocas no cierran por su parte superior.

25 Iniciales con pie dividido en precarolina alemana del s. IX (Lorsch), en Ángel CANELlas, Exempla scripturarum latinarum, I, Zaragoza 1963, XXXVII. 
$p q$ Caído inclinado hacia la izquierda y ojo a veces sin cerrar.

$r$ Muy inclinada. Trazo final se alarga en fin de palabra. En comentarios se desvirtúa su forma debido a la dificultad de trazarla en módulo pequeño; su caído se alarga en ocasiones bajo la línea de renglón (narrantur, primera columna, cara carne).

$s$ Algo inclinada a la izquierda.

$t$ De dos tipos, en ambos con la línea superior horizontal. Toca las letras anterior y posterior por su trazo superior. En comentarios su trazo final se alarga en fin de palabra. Forma algún nexo (ty en cara carne).

$x$ Aspada. El primer trazo sobrepasa la línea de renglón.

$y$ No aparece en cara pilosa. En la opuesta, una sola vez en el centro (nexo $t y$, sin punto) y varias en comentarios (con punto).

$z$ Un solo ejemplo en Zelotes, columna central. Forma carolina normal con pequeño copete y trazo inferior recto totalmente.

$b d b l \mathrm{El}$ astil en ocasiones algo más grueso en su parte superior $\mathrm{e}$ inclinado a la derecha. De altura variable.

$a c l r$ Tocan con frecuencia la letra siguiente (láminas 6 y $7{ }^{26}$ ).

NeXOS.- Son abundantes aunque en la mayoría de los casos consisten únicamente en letras que se tocan sin modificar su forma originaria. No es éste el caso de et st ct (este último aparece una sola vez, en los comentarios), procedentes de las cursivas, de ty ex y de la e caudata ${ }^{27}$ (lámina 8).

ABREVIATURAS. - En el lado pelo el signo general abreviativo es la raya que aparece abreviando $m$ en tuam duodecim (texto central), bonorum (comentarios); $n$ en iubent (comentarios); en nomina sacra: Ihs I hm di (texto central), dnm $d m$ xpi (comentarios); raya sobre $e=e s t$, columna derecha. En el lado carne no aparece ninguna abreviatura en el texto principal. En comentarios la raya como signo general abrevia $m$ en multitudinem y aparece de forma abundante en nomina sacra: dni dno dns xpm xpo di sps.

OTROS SIGNOS.- Pueden verse los de puntuación, que se usan sin pauta regular, en la lámina 9, junto con los signos de llamada. A cada uno de estos últimos corresponde, en las columnas de comentarios, un párrafo al final de cual hay punto y aparte. En el lado piloso aparecen siete signos en el texto

20 Dado el escaso tamaño de las letras de los comentarios, reproduzco el alfabeto a través de palabras completas.

" Los citados, entre otros, son típicos de la segunda década del s. IX: B. BisCHOFF, Eine Sammelhandschrift Walafrid Strabos, Mittelalterliche Studien, II, pp. 38. 
principal. En las columnas laterales, en las que se ve uno ausente en el centro, no pueden apreciarse los demás debido a las manchas del pergamino. En el lado carne la primera columna está mutilada, por lo que se han perdido todas sus llamadas. En el centro se ven cinco signos, dos de los cuales se repiten en la columna tercera. En esta cara se ven en total siete párrafos, por lo que no son visibles al menos dos signos. Algunos de éstos pudieran estar inspirados en grafías insulares.

Por último, aparecen algunas correcciones de omisiones que se solucionan mediante interlineado de las letras olvidadas.

Siguen transcripción y láminas anunciadas en el texto. ${ }^{28}$

\author{
CARLOS SÁEZ \\ Universidad Complutense \\ Alcalá de Henares
}

\title{
TRANSCRIPCIÓN DEL TEXTO CENTRAL
}

LUCAS 6, 8-19

$8 \quad \begin{aligned} & \text { (lado pelo) } \\ & \text { [homini, qui habebat] } \\ & \text { manum aridam: } \\ & \text { "Surge et sta in me- } \\ & \text { dium». Et surgens stetit. } \\ & \text { Ait autem ad illos Ihesus: } \\ & \text { "Interrogo uos, si licet } \\ & \text { sabbatis bene facere } \\ & \text { an male, animam sal- } \\ & \text { uam facere an perde- } \\ & \text { re». Et circumspectis } \\ & \text { omnibus dixit homini: } \\ & \text { "Extende manum tuam». } \\ & \text { Et extendit et restitu- } \\ & \text { ta est manus eius. } \\ & \text { Ipsi autem repleti sunt } \\ & \text { insipientia, et conlo- } \\ & \text { quebantur ad inuice } \\ & \text { quid nam facerent } \\ & \text { Ihesum. } \\ & \text { Factum est autem in } \\ & \text { illis diebus exiit in }\end{aligned}$

28 Dejo la transcripción de los comentarios para otra ocasión por evitar la excesiva extensión del trabajo.

${ }^{29}$ Puede verse la parte inferior de la línea. La reconstruyo junto con otras dos más adelante según la versión de José O'CALLAGHAN-José María BOVER, Nuevo testamento trilingüe, BAC, Madrid 1977, pp. 332-334. 
montem orare, et erat pernoctans in oratione Dei.

13 Et cum dies factus esset, uocauit discipulos suos, et elegit duodecim ex ipsis

(lado carne)

[quos et apostolos nominavit: ${ }^{30}$

14 Simonem quem et cog- ${ }^{31}$ nominauit Petrum, et Andream, fratrem eius, Iacobum et Iohannem, Philippum et Bartholo-

15 meum, Mattheum et Thomam, Iacobum Alphaei et Simonem, qui uo-

16 catur Zelotes, Iudam Iacobi et Iudam Scarioth, qui fuit proditor.

17 Et descendens cum illis, stet in loco campestri, et turba discipulorum eius et multitudo copiosa plebis ab omni Iudaea et Hierusalem, et maritima et Tyrii, et Sidoni qui uenerant ut audirent eum, et sanarentur a langoribus suis: et qui uexabantur ab spiritibus inmun-

19 dis curabantur. Et omnis turba quaerebat eum tangere quia uirtus de illo exiebat et sanabat omnes.

\section{RÉSUMÉ}

La coutume médiévale de ré-utilisation de vieux manuscrits nous a légué un nombre élevé de restes de livres aujourd'hui disparus, qui sont des témoignages de grande valeur sur notre passé. Tel est le cas d'un parchemin, feuille indépendante qui est apparue, collée à la couverture de bois d'un manuscrit musical ancien, du XIVème siècle. Le folio est composé de trois colonnes et contient un fragment de l'Évangile de Saint Luc (Luc 6, 8-19) au centre, et de commentaires du texte principal de part et d'autre. Ces commentaires sont accompagnés de signes de repaire qui se répètent dans la colonne centrale aux points correspondants. D'après l'écriture et la disposition du texte on peut dater cet exemple du début du IXème

30 Esta línea falta por completo.

3 Puede verse la parte inferior de la línea. 
siècle, dans sa deuxième ou troisième décennie et on peut supposer qu'il vient de l'ouest de l'Allemagne. Le plus ancien exemplaire commenté de l'époque carolingienne qui nous soit parvenu provient d'Irlande, il date du VIIIème siècle et contient un texte principal d'Ezéquiel et comme commentaire une célèbre homélie de Grégoire. L'exemple qui est présenté ici est l'un des premiers connus de la Bible carolingienne commentée.

\section{SUMMARY}

The medieval habit of reusing the old manuscripts provides us with a large number of remains of books that have disappeared nowadays but that constitute testimonies of great value about our past. It is the case of one independent folio of parchment that came up, stuck on the wooden cover of a musical codex dating from the XIV ${ }^{\text {th }}$ century. The folio is divided into three columns and contains a piece of Saint Luke 's Gospel (Luke 6, 8-19) in the middle, and notes on the original text in the margins. There are also calling signs repeated in the central column just where they fit. According to its script and disposition, the text of this example could be dated from the principle of the $\mathrm{IX}^{\text {th }}$ century, in its second or third decade, and comes probably from West Germany. The best conserved specimen of the Carolingian time kept nowadays comes from Ireland and is dated from the VII ${ }^{\text {th }}$ century. It contains the main text of Ezequiel and, as notes, fragments of a famous homily by Gregory. The above presented specimen is one of the first of the Carolingian commented Bible. 


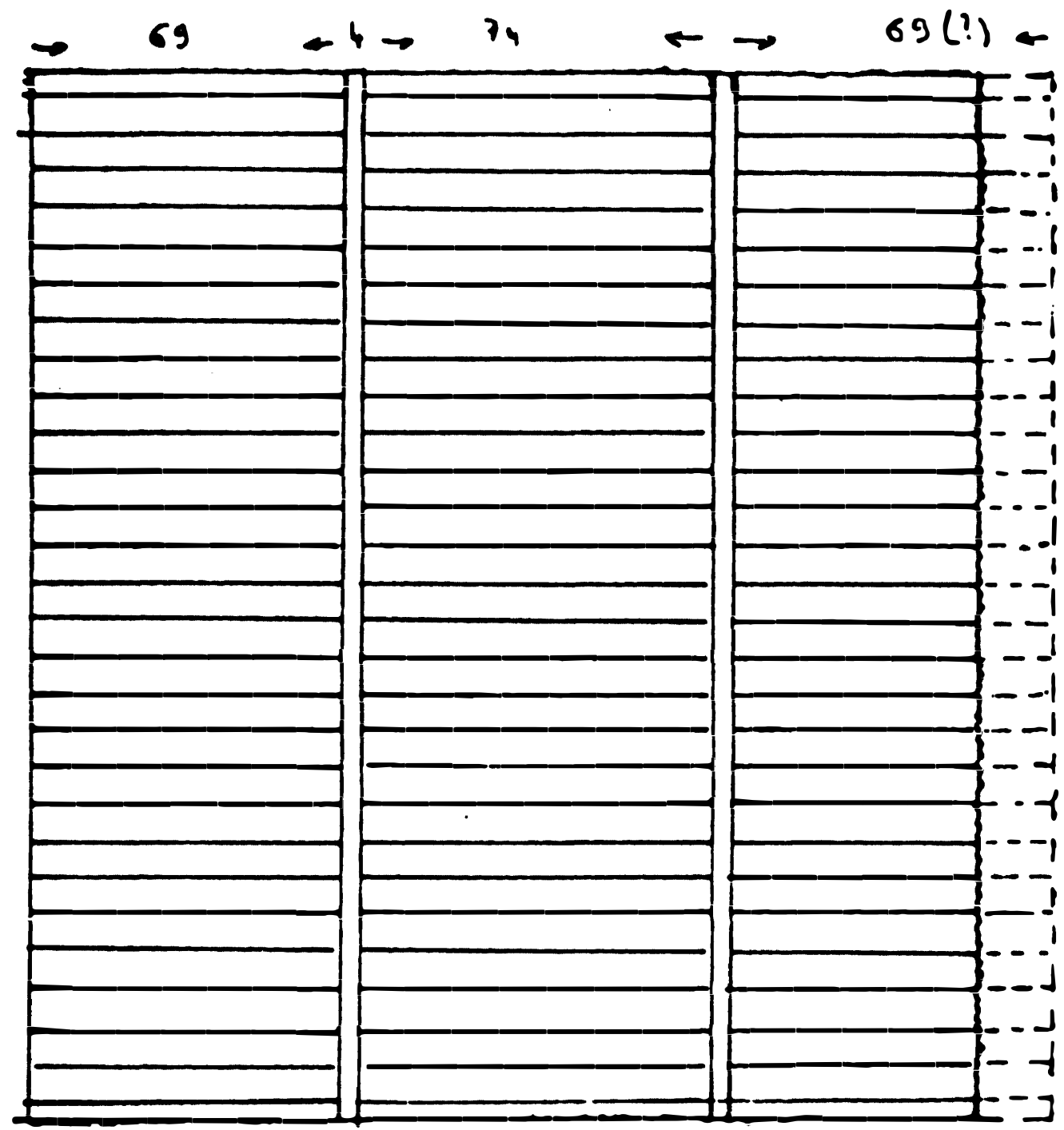

Fig. 1. Zürich, Staatsarchiv, AG 19 N. ${ }^{\circ}$ XII, s. VIII/IX Irlanda (CLA VIII 1008); HOLTZ, PL. 1 izq. 


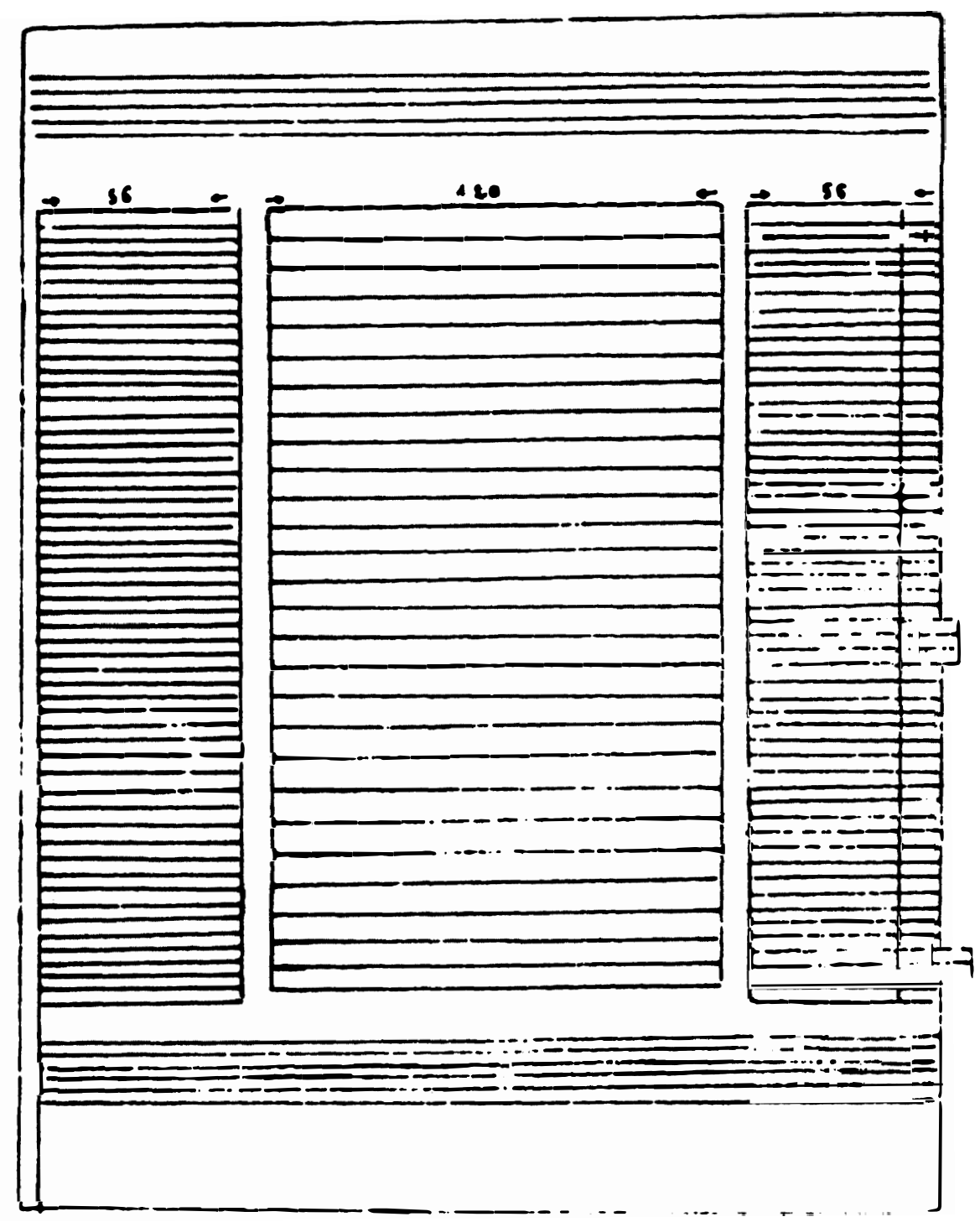

Fig. 2. Bern, Burgerbibliothek 165, s. IX' Tours; HOLTZ, PL. 2 izq. 


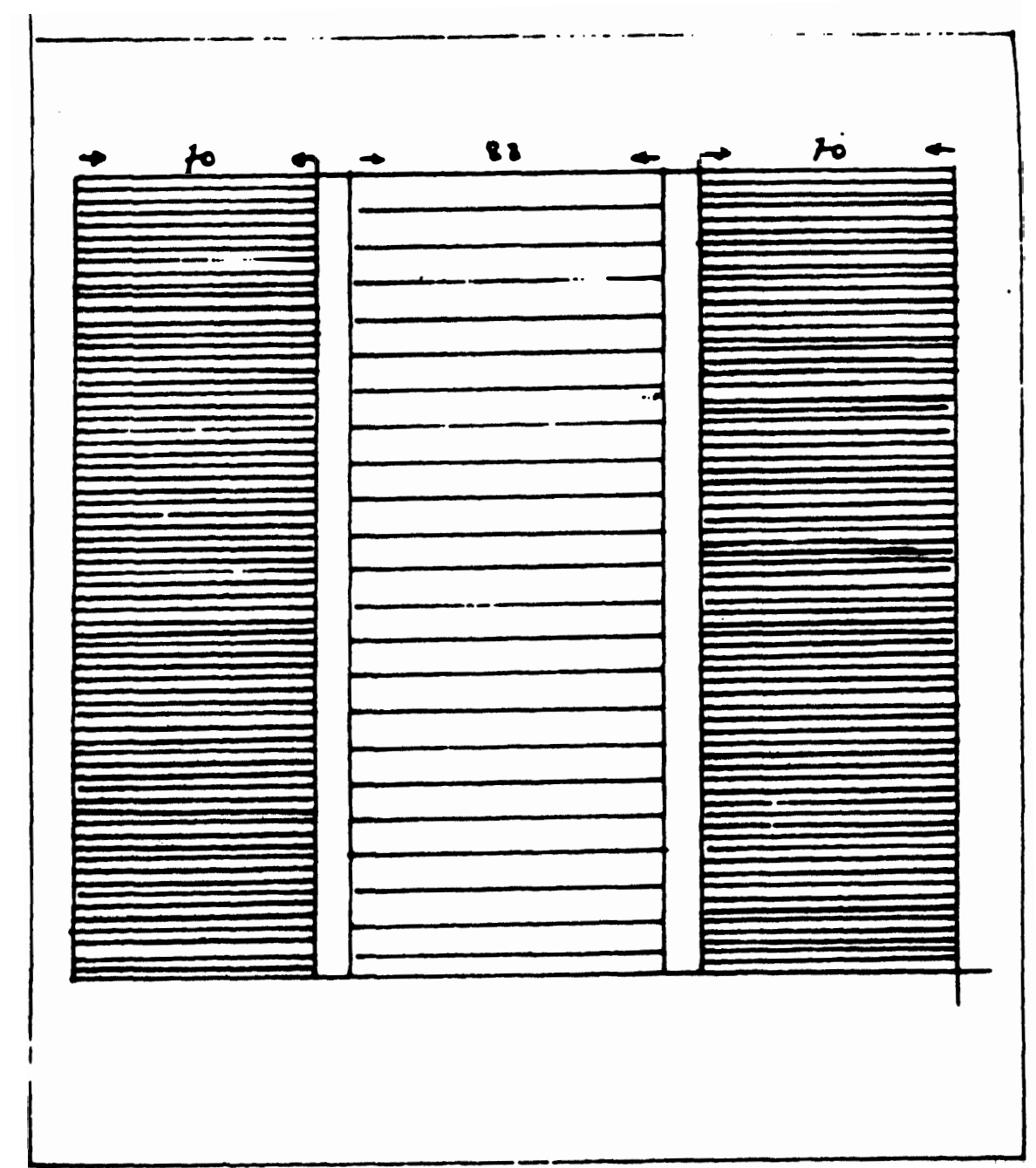

Fig. 3. Bern, Burgerbibliothek 172, s. IX ${ }^{\mathrm{m}}$ Fleury; HOLTZ, PL. 1 dcha. 


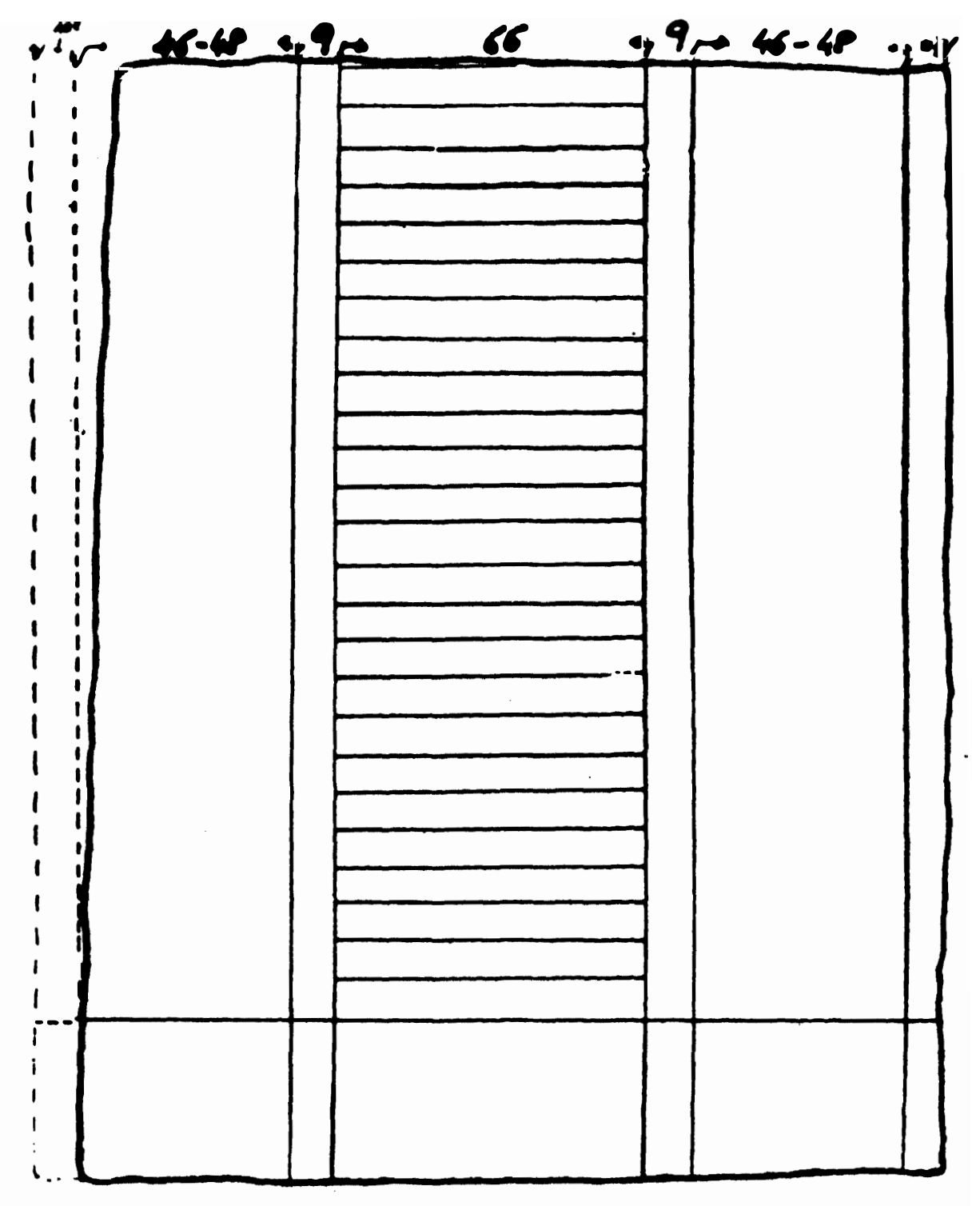

Fig. 4. Ejemplo estudiado. 

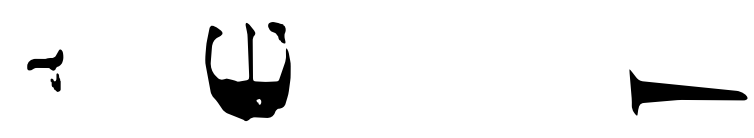

2
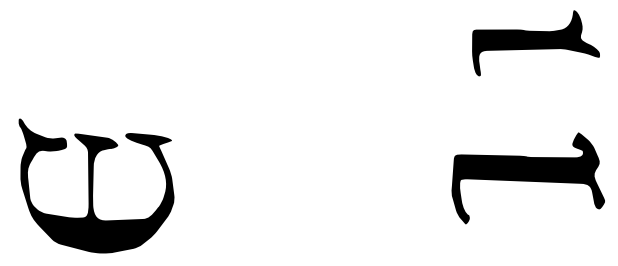

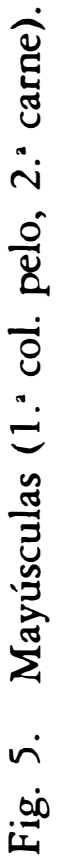
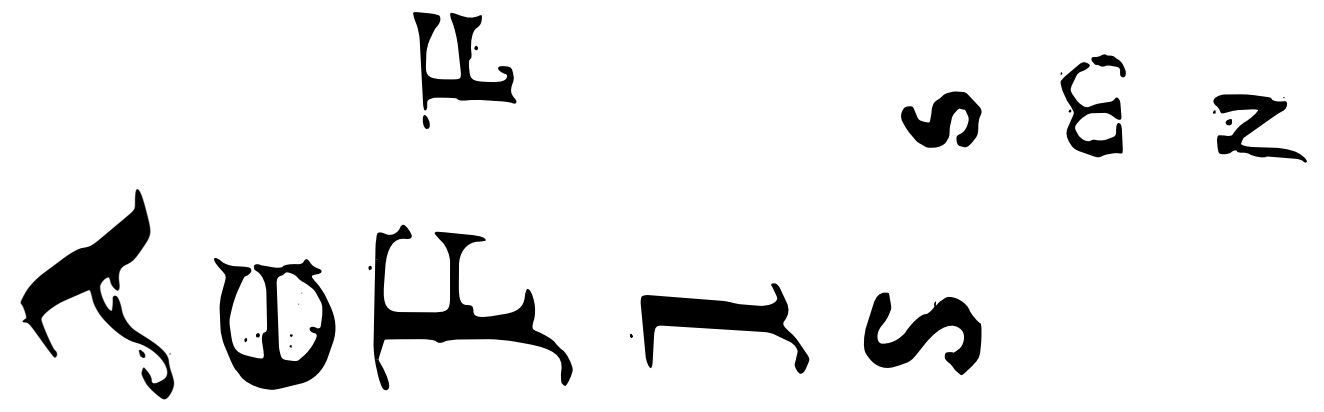


\section{$m$ m}

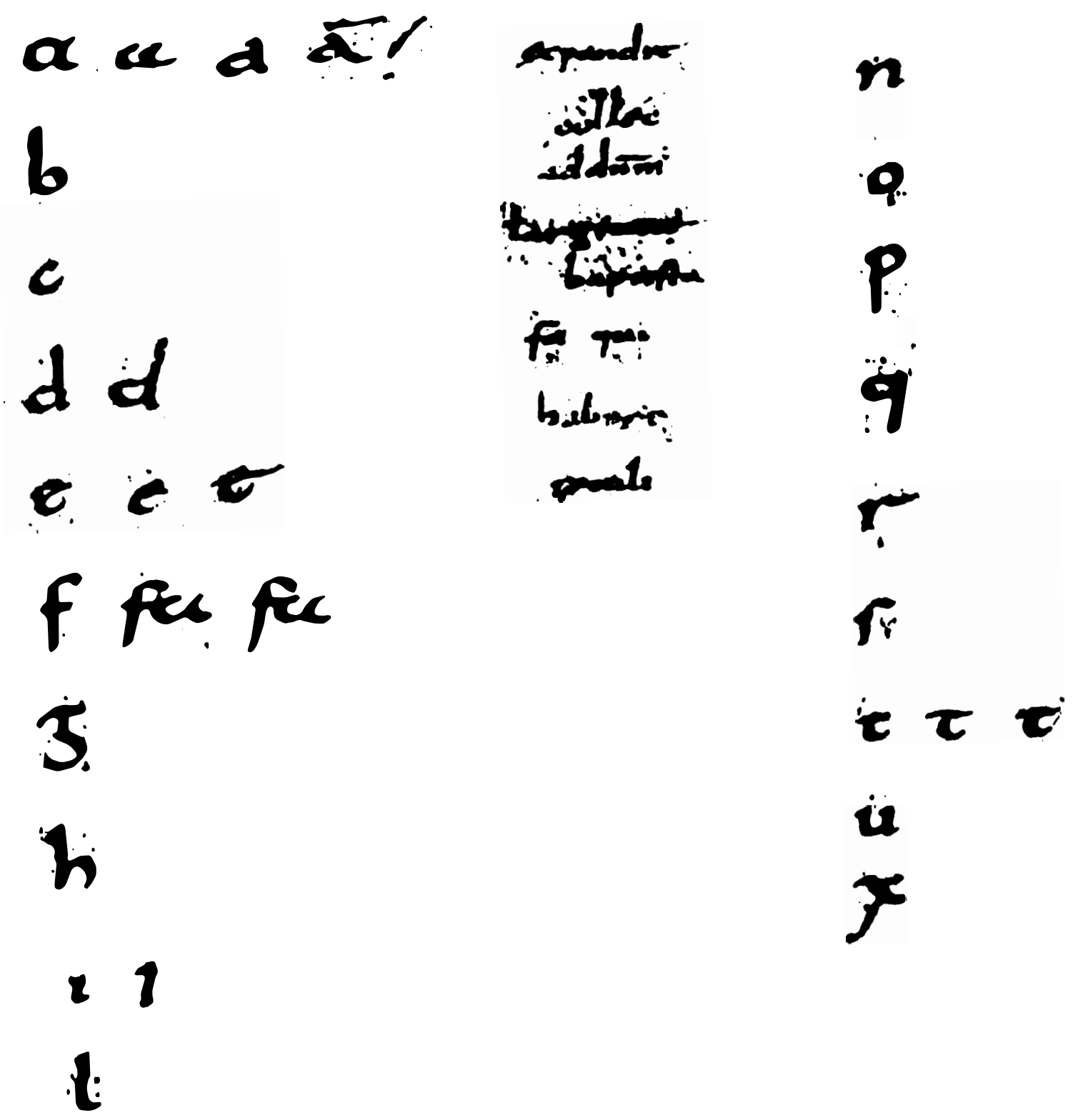

Fig. 6. Minúsculas, lado pelo. 


\section{$m m$ m}

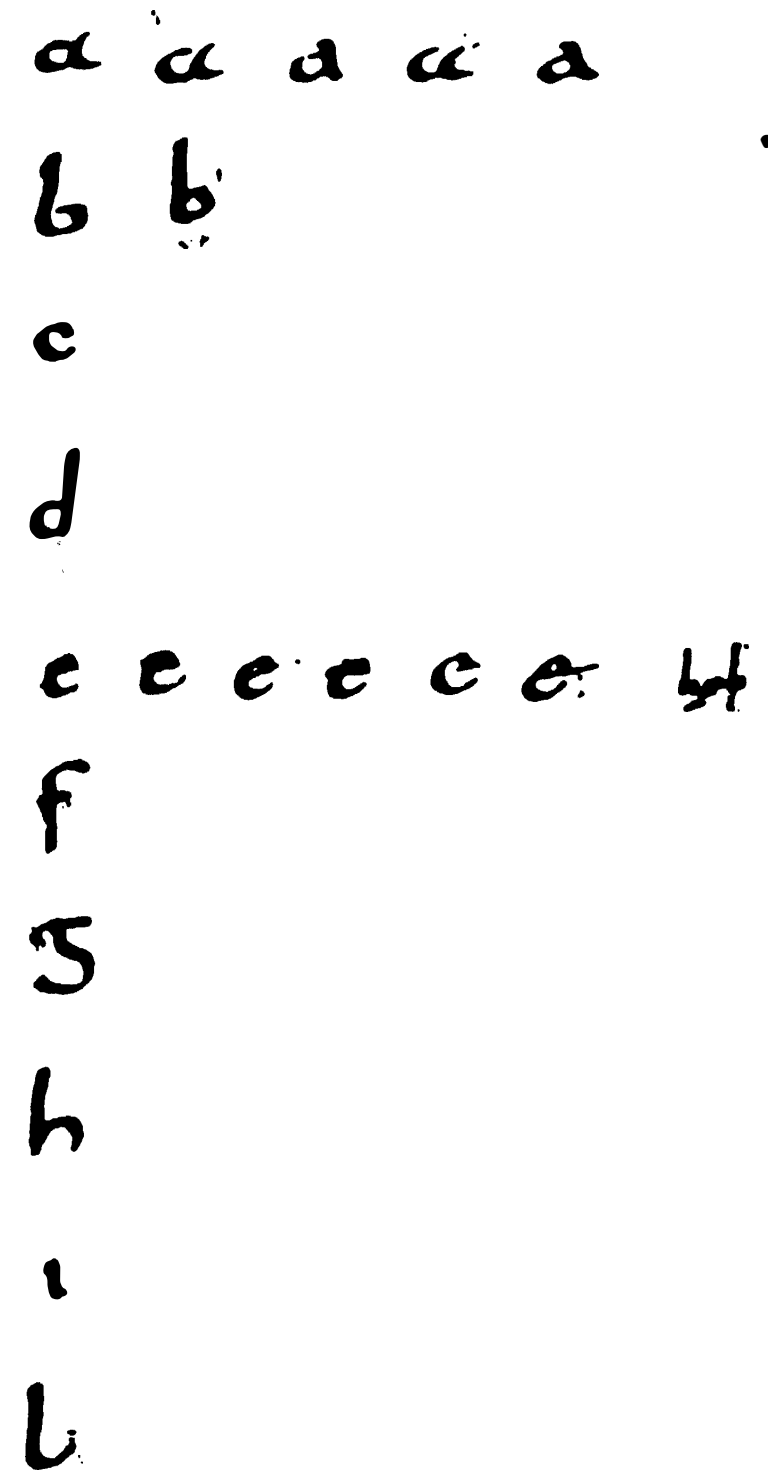

$n n$

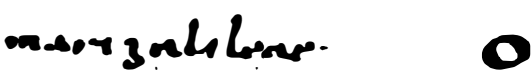

nispare dapo

encobir bo

$P \rho$

rymar fi

9

$r$
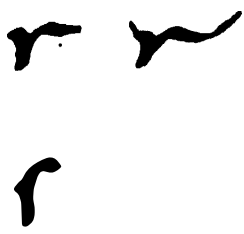

2

$\boldsymbol{u}$

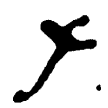

$\checkmark$

5

Fig. 7. Minúsculas, lado carne. 


$$
-8
$$
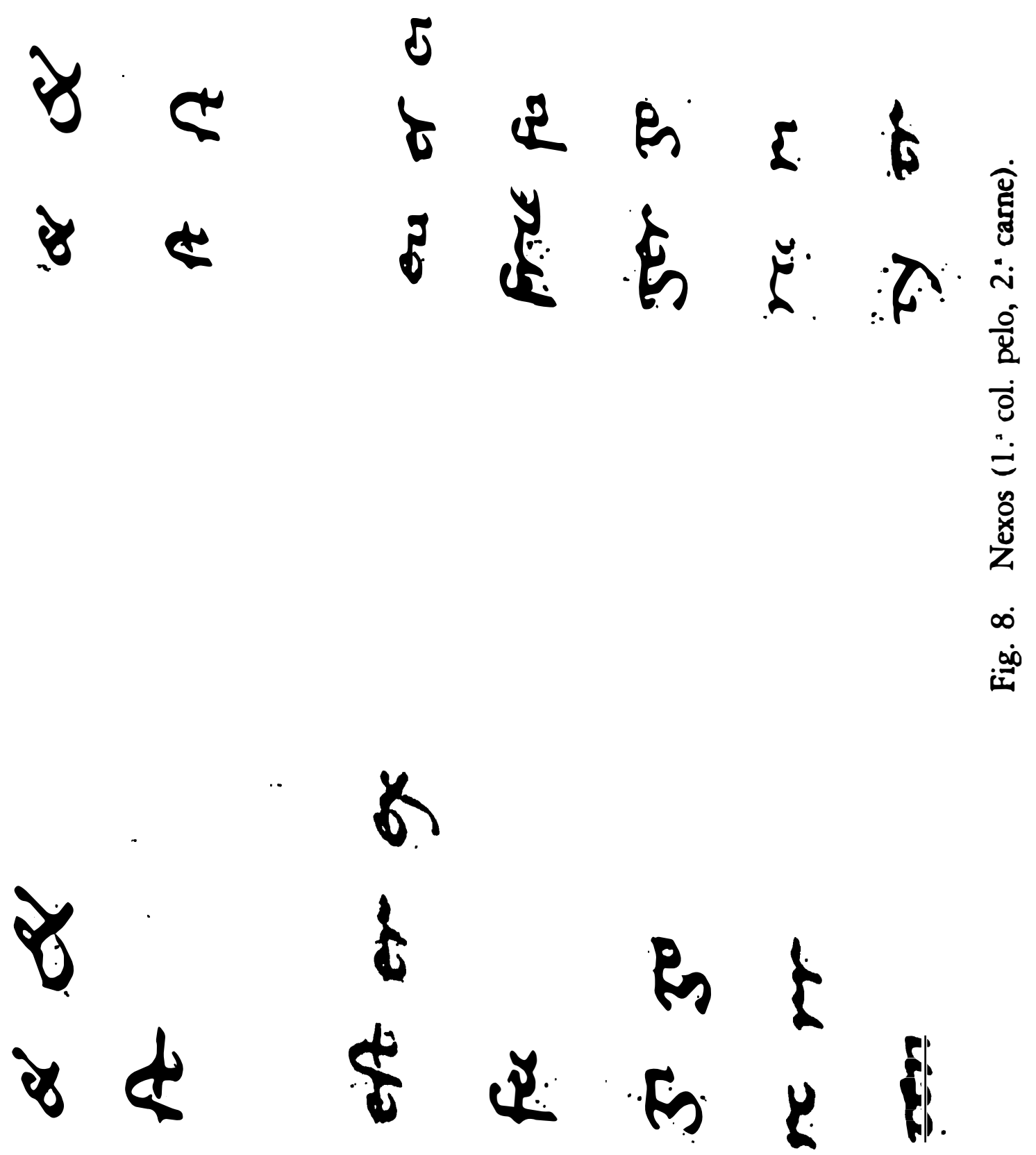

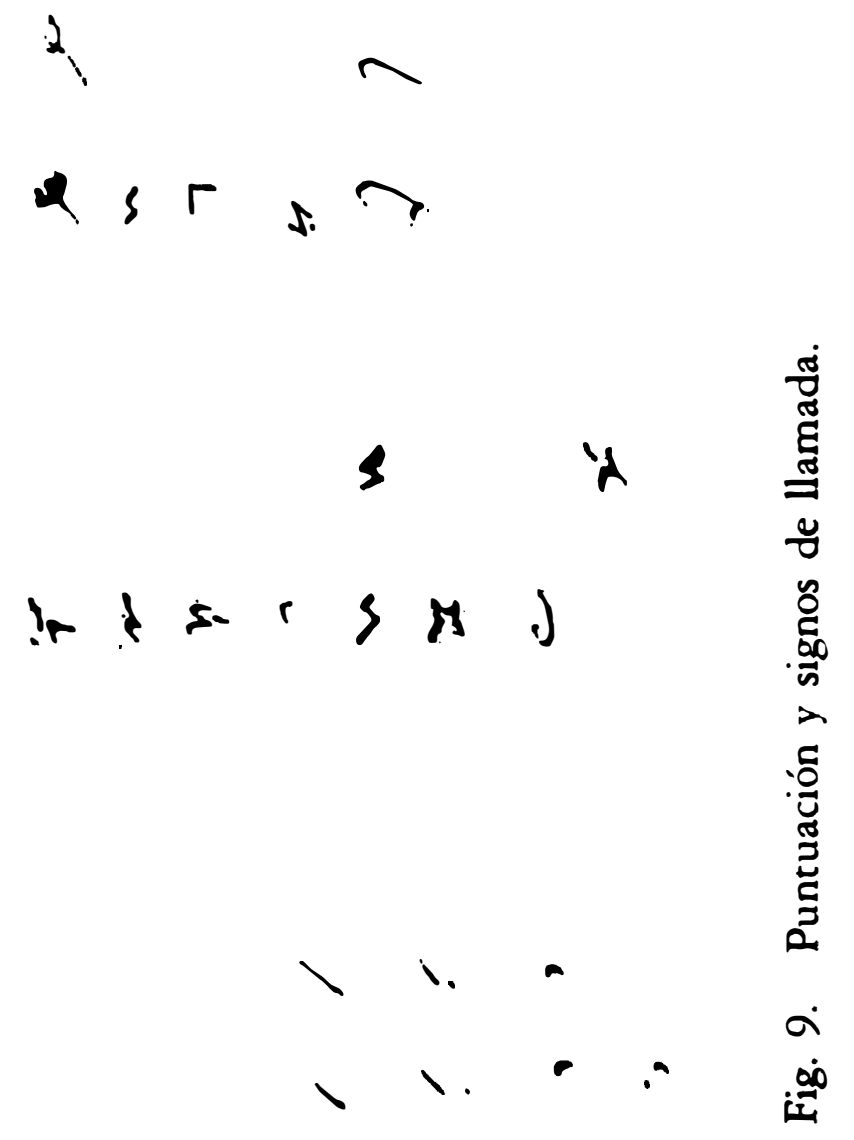


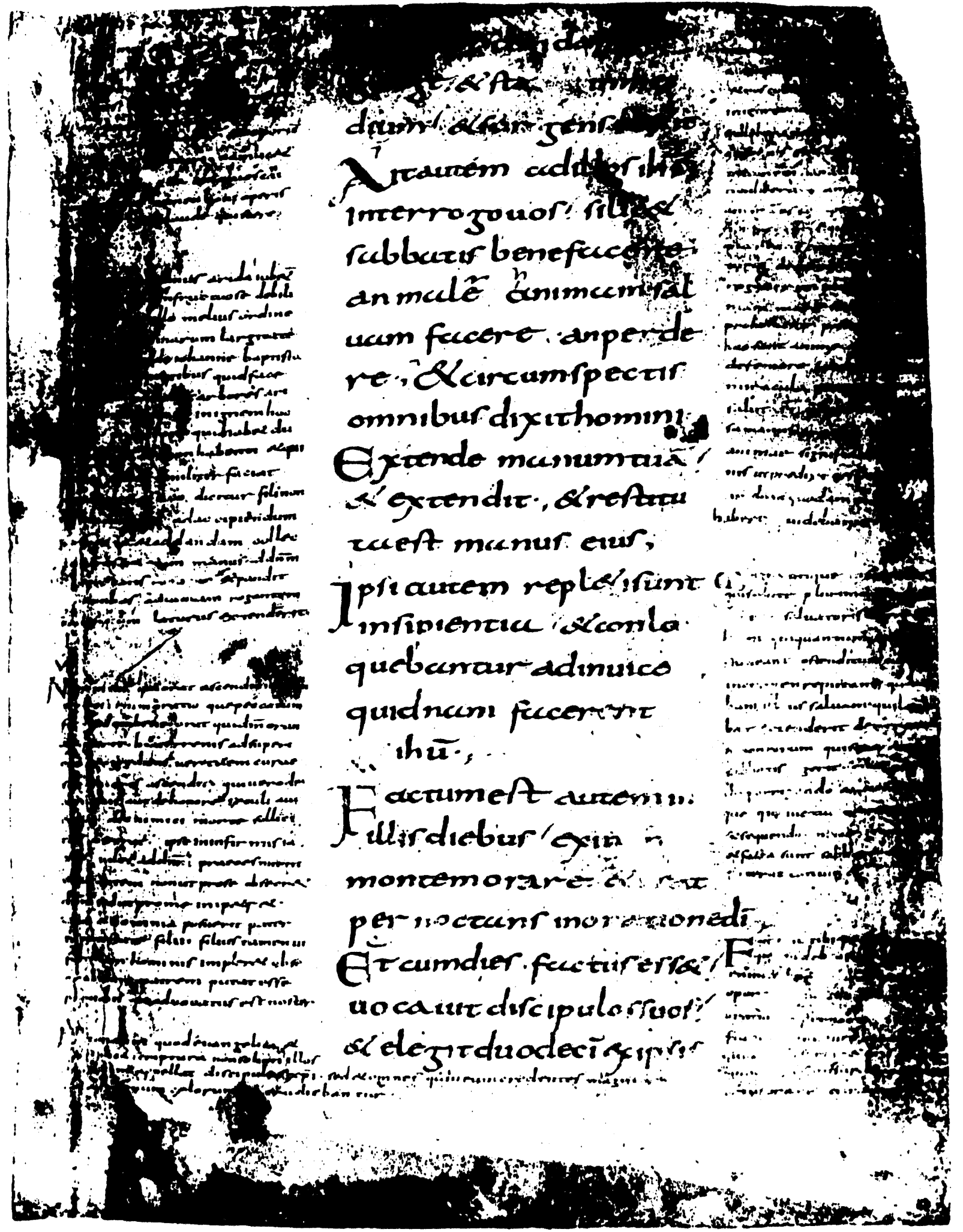

Fig. 10. Lado pelo. 


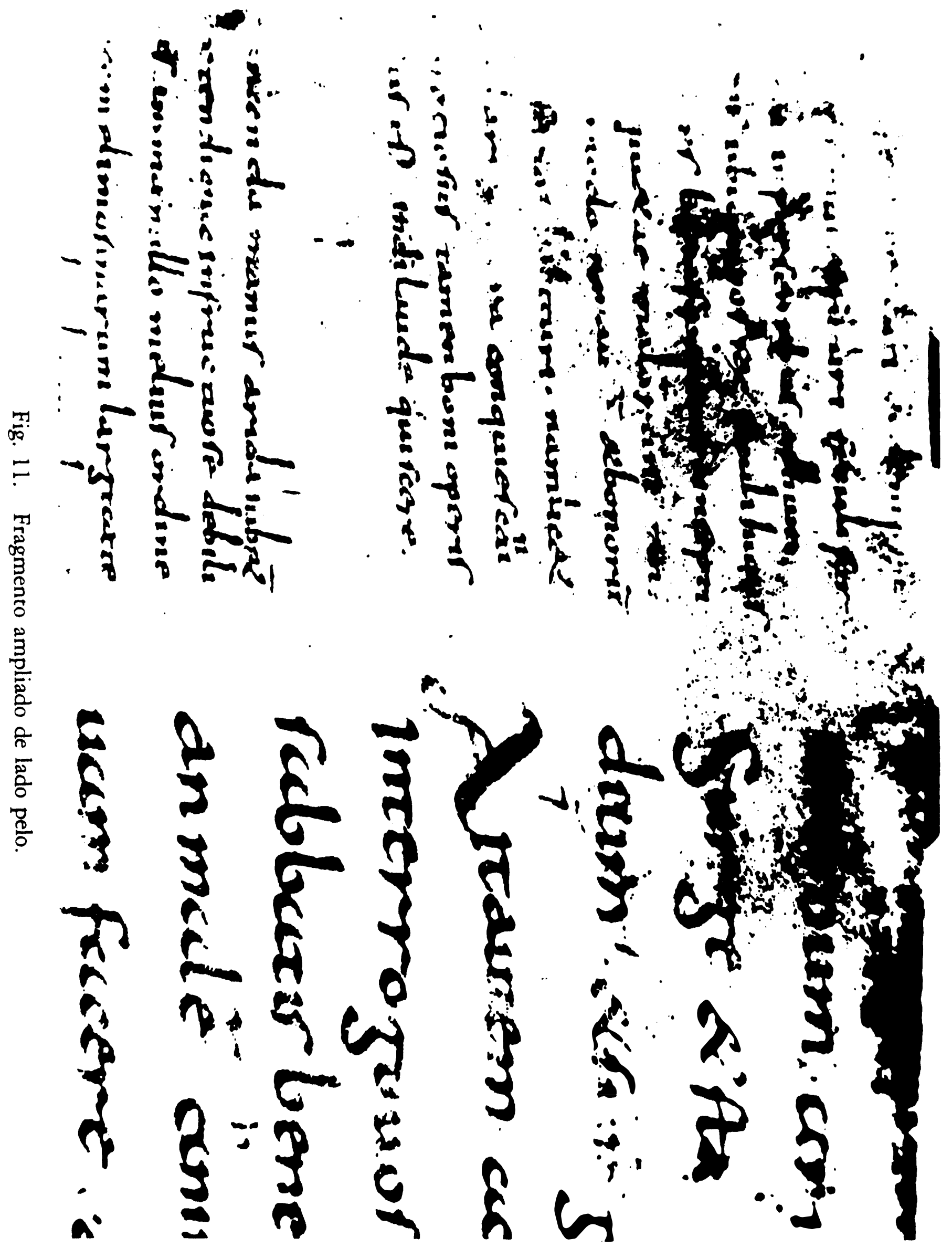




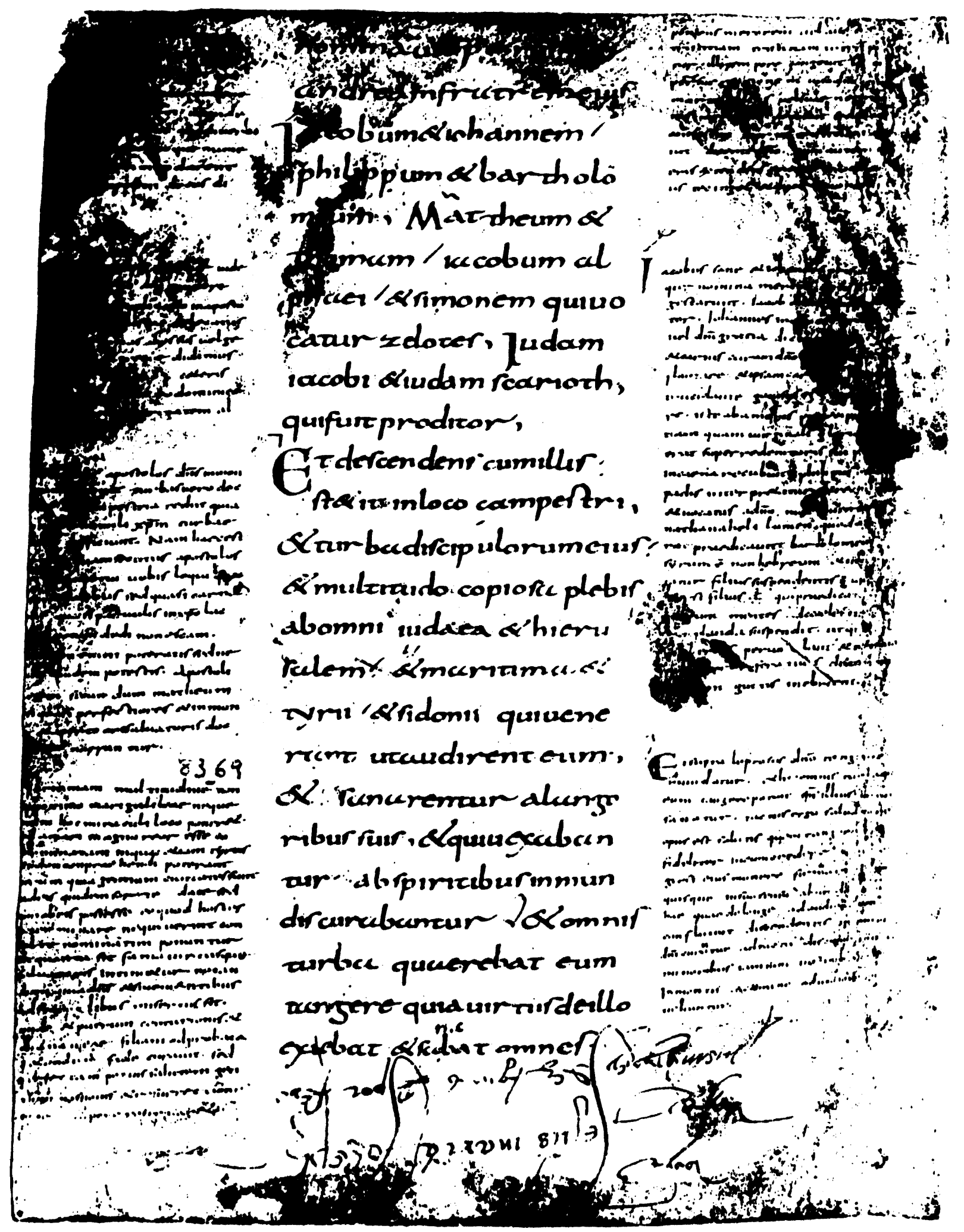

Fig. 12. Lado carne. 


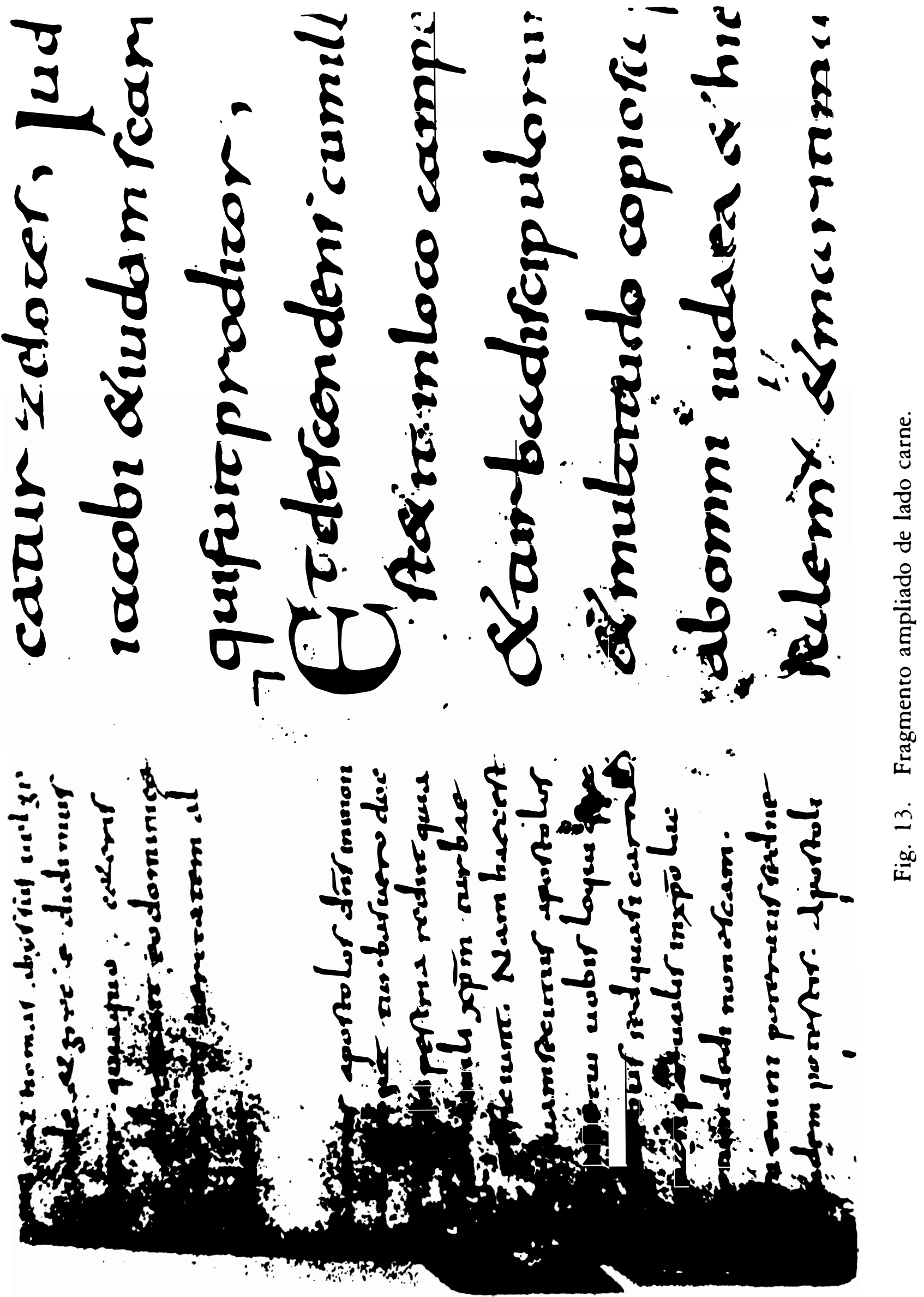

